\title{
Steingarten, Schweizerhaus, Hängebrücke und Wasserfall
}

\author{
Hilke Steinecke \& Peter Schubert
}
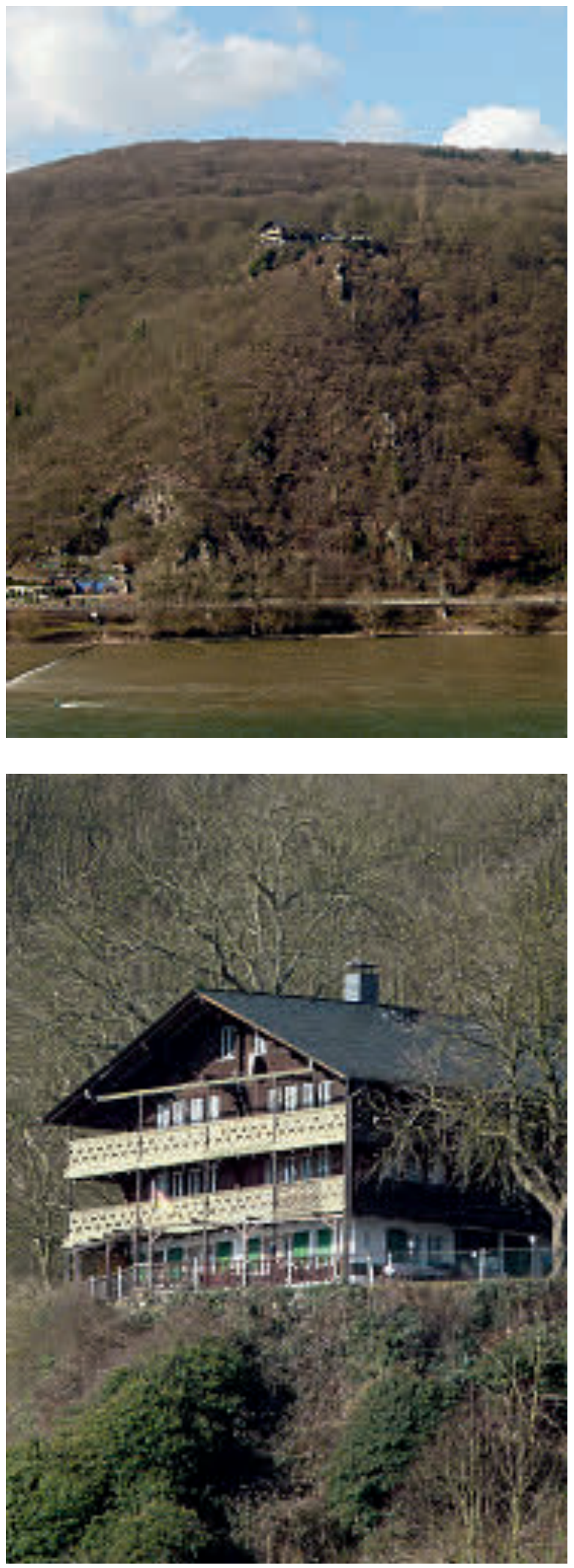

\section{Idealbild einer romantischen Landschaft}

Ende des 18. bis weit ins 19. Jahrhundert war die Schweizer Bergwelt das Idealbild einer Landschaft. Deshalb war die Schweiz auch eine Station der legendären Grand Tour. Hierunter versteht man seit der Renaissance eine obligatorische Bildungsreise (Angehöriger des europäischen Adels und später ebenso des gehobenen Bürgertums) durch Mitteleuropa. Ziele waren vor allem Italien, Spanien, aber auch die Schweiz und das Heilige Land. Die Grand Tour spiegelte sich im 18. Jh. vor allem in England in vielen literarischen Werken wider.

Man begeisterte sich in der Schweiz für erhabene Berge, rauschende Wasserfälle, Seen und nicht zuletzt für die Menschen und ihre Behausungen. Die Gehöfte der schweizerischen Bergbevölkerung waren symbolträchtig, da sie Teile der wildromantischen Landschaft darstellten. Die Schweiz und ihre Bewohner versinnbildlichten zudem das freiheitliche Denken in der ersten Demokratie Europas. In ihrer Heimat zurück, ließen sich die Teilnehmer einer Grand Tour in ihren Parks oft Steingärten anlegen und zuweilen auch ein "Schweizerhaus" bauen, um ihre Naturbegeisterung und ihre freiheitsliebende Gesinnung zum Ausdruck zu bringen.

\section{Schweizerhäuser}

Im Mittelrheintal bei Trechtinghausen nutzte man 1842-1844 die natürlichen Steilhänge, um auf einem Felsplateau in $130 \mathrm{~m}$ Höhe über dem Rheintal ein Schweizerhaus zu errichten,

Abb. 1 (oben): Das Schweizerhaus am Rhein bei Trechtingshausen.

Abb. 2 (unten): Das Schweizerhaus bei Trechtingshausen aus der Nähe, März 2015.

Abb. 3 (Seite 65 oben): Das Schweizerhaus im Palmengarten auf einer alten Ansichtskarte.

Abb. 4 (Seite 65 unten): Blick vom Rhododendrongarten über den Weiher auf den Wasserfall, Mai 2014. 

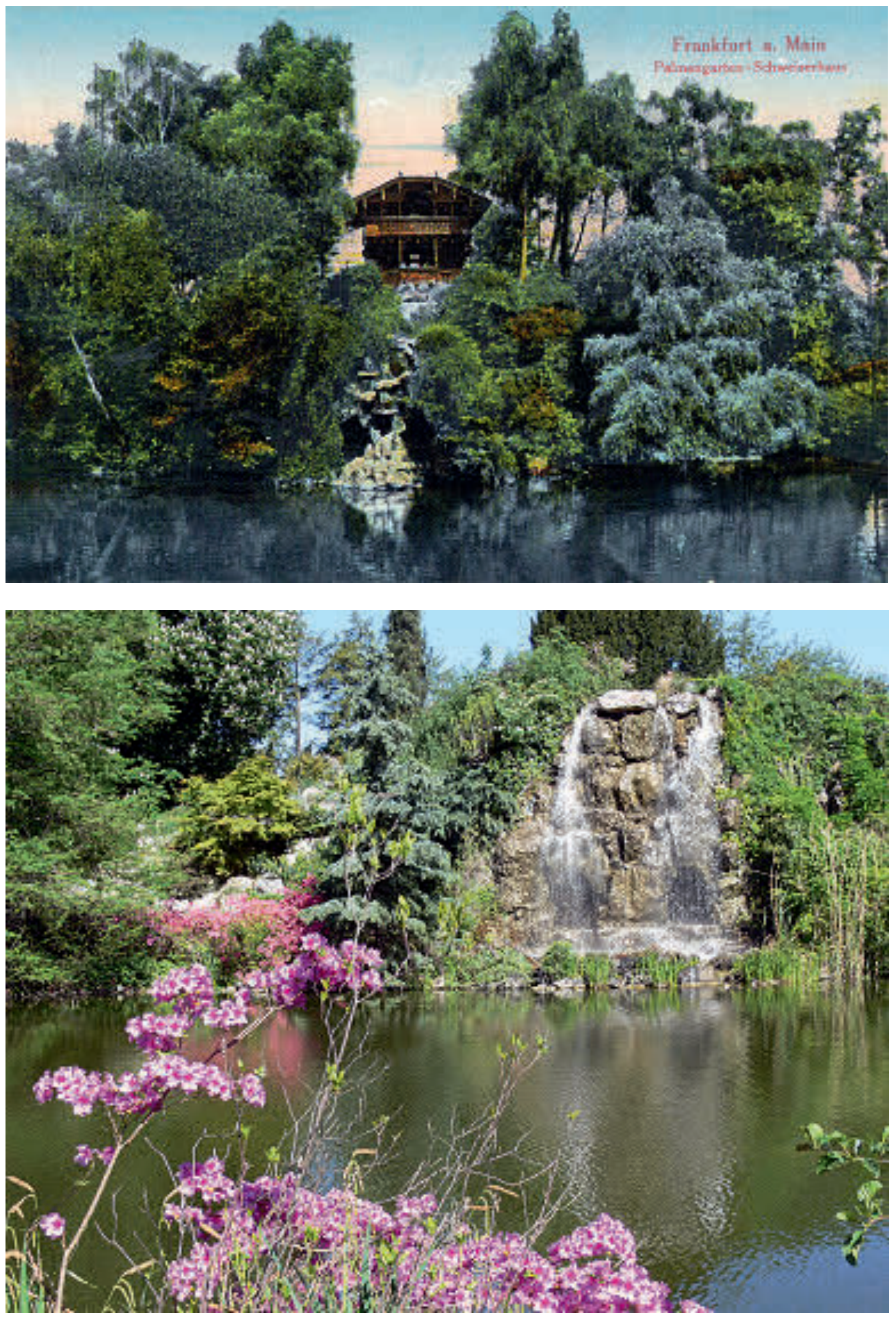


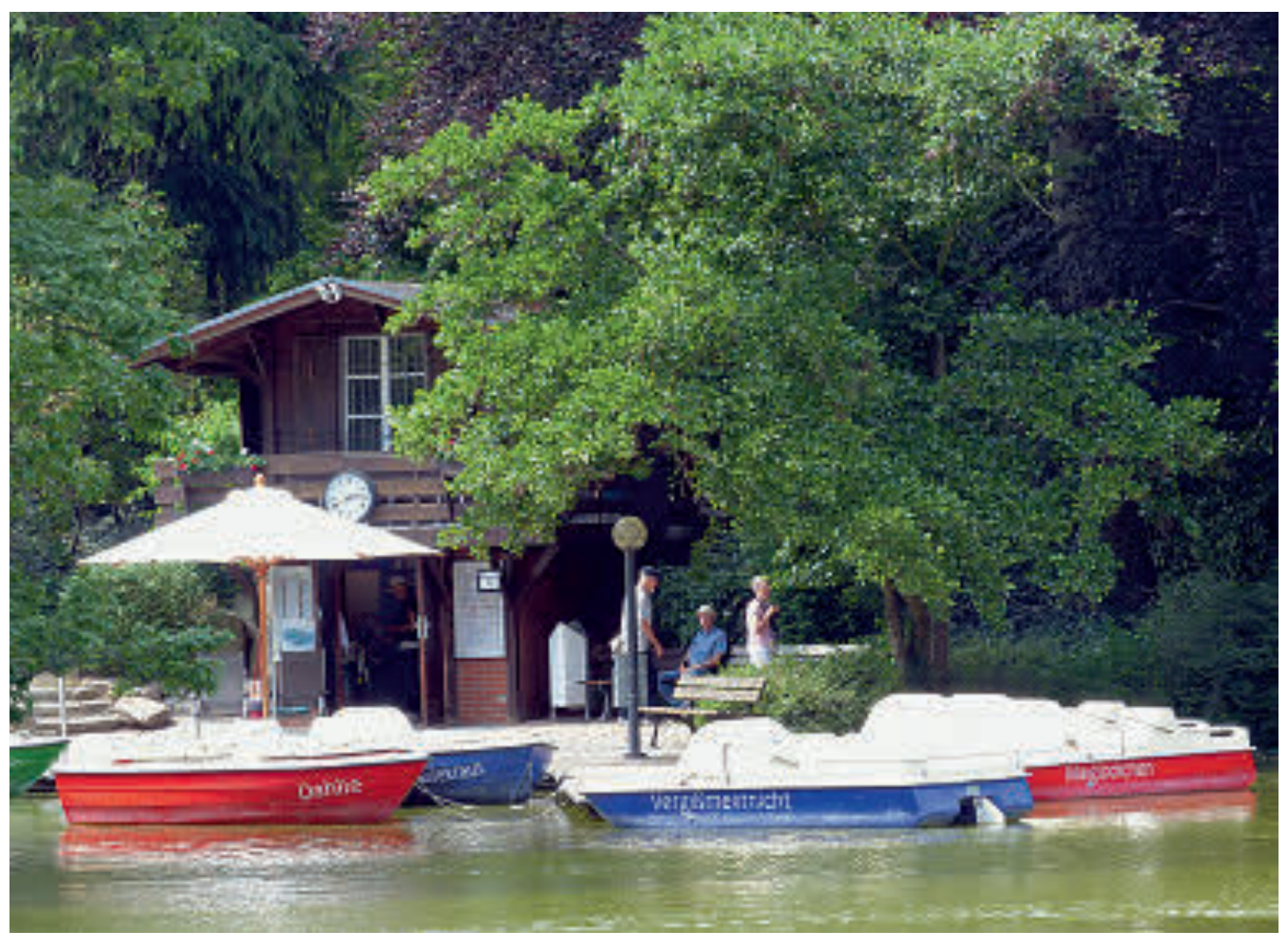

das noch heute steht. Es diente Prinz FriedRich Wilhelm Ludwig von Preußen als Jagdhaus und Unterkunft seiner Gäste, wenn er auf Schloss Sooneck weilte. Schweizerhäuser wurden allerdings nicht immer ganz vorbildgetreu nachgebaut. Wegen der großen Popularität der Schweizerhäuser wurden sie ab der 2. Hälfte des 19. Jahrhunderts sogar fabrikmäßig hergestellt. Auf der Pariser Weltausstellung wurden 1867 „Swiss Chalets“ als Fertighäuser vorgestellt.

\section{Das Schweizerhaus im Palmengarten}

Auch im Palmengarten gibt es ein Stückchen Schweiz. Bei der Anlage des großen Weihers fiel Aushub an, den Heinrich Siesmayer zur Einrichtung eines Steingartens nutzte. Den Steingarten und auch die Grotte, die sich innerhalb des Hügels befindet, genauer durchzieht, gibt es noch heute. Die Grotte diente sicherlich schon vielen (verliebten) Palmengartenbesuchern als ein lauschiger romantischer Rückzugsort. Das Schweizerhaus befand sich auf dem höchsten
Punkt des Steingartens. Oft ist es überhöht dargestellt, um seine Lage noch schroffer und wilder erscheinen zu lassen. Es wurde 1931 wegen Baufälligkeit abgerissen und entsprach auch nicht mehr dem Zeitgeschmack. Auf alten Abbildungen kann man es allerdings noch bewundern. Das Bootshaus, das nunmehr in dritter Generation am Großen Weiher steht, zeigt ebenfalls den Stil eines Schweizerhauses.

\section{Hängebrücke}

Über den Weiher führte ursprünglich eine Kettenbrücke, die 1874 von Siesmayer errichtet

Abb. 5 (oben): Das Bootshaus ist ebenfalls im Stil eines Schweizerhauses errichtet, Mai 2015. Es gibt aktuell Ruder- sowie Tretboote, die jeweils den Namen einer Blume tragen.

Abb. 6 (Seite 67 oben): Blick über den Bootsweiher mit Bootshaus, Fontäne, Hängebrücke und Wasserfall auf einer alten Ansichtskarte.

Abb. 7 (Seite 67 unten): Besucher am Bootsweiher mit Blick auf das Bootshaus auf einer alten Ansichtskarte. 

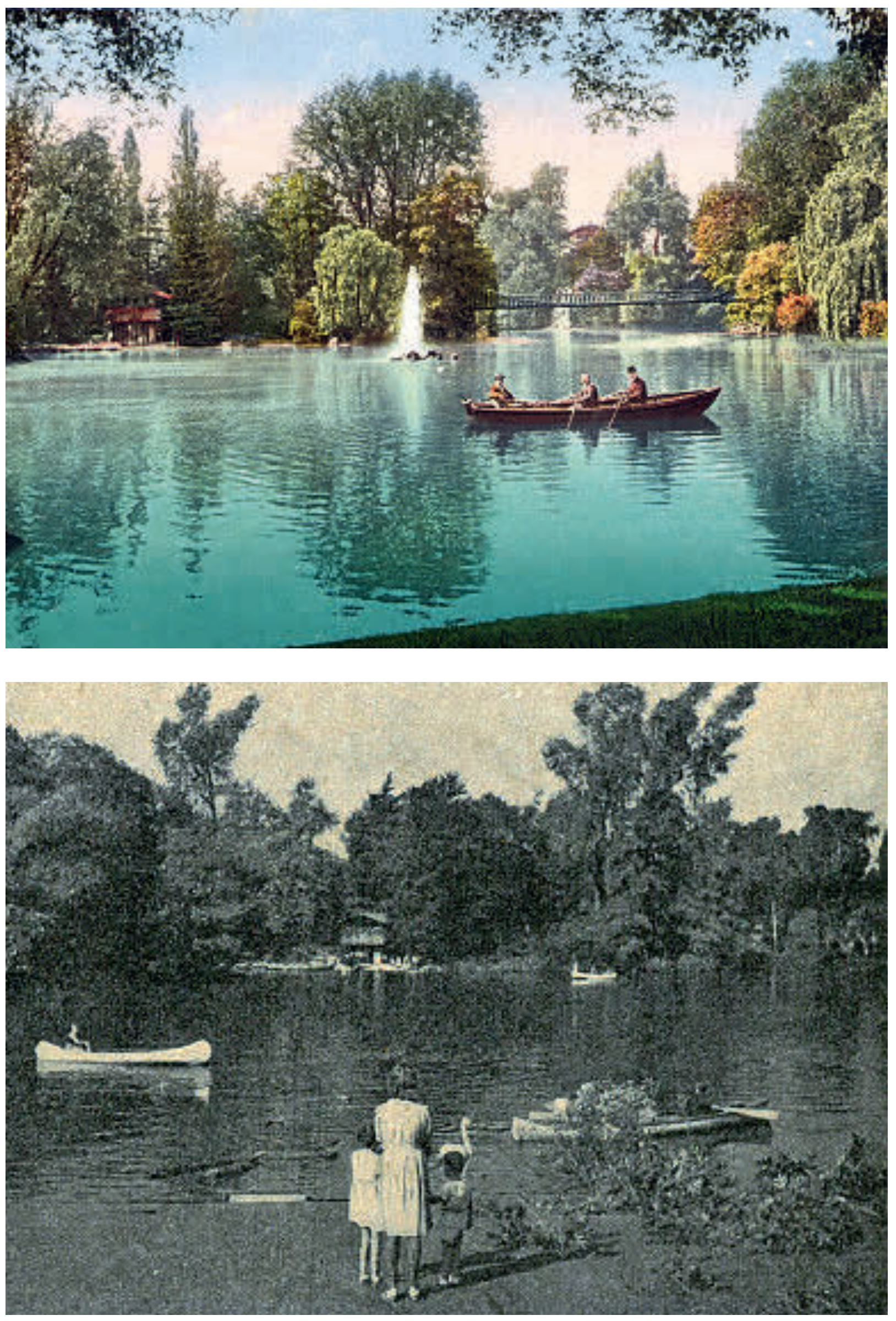




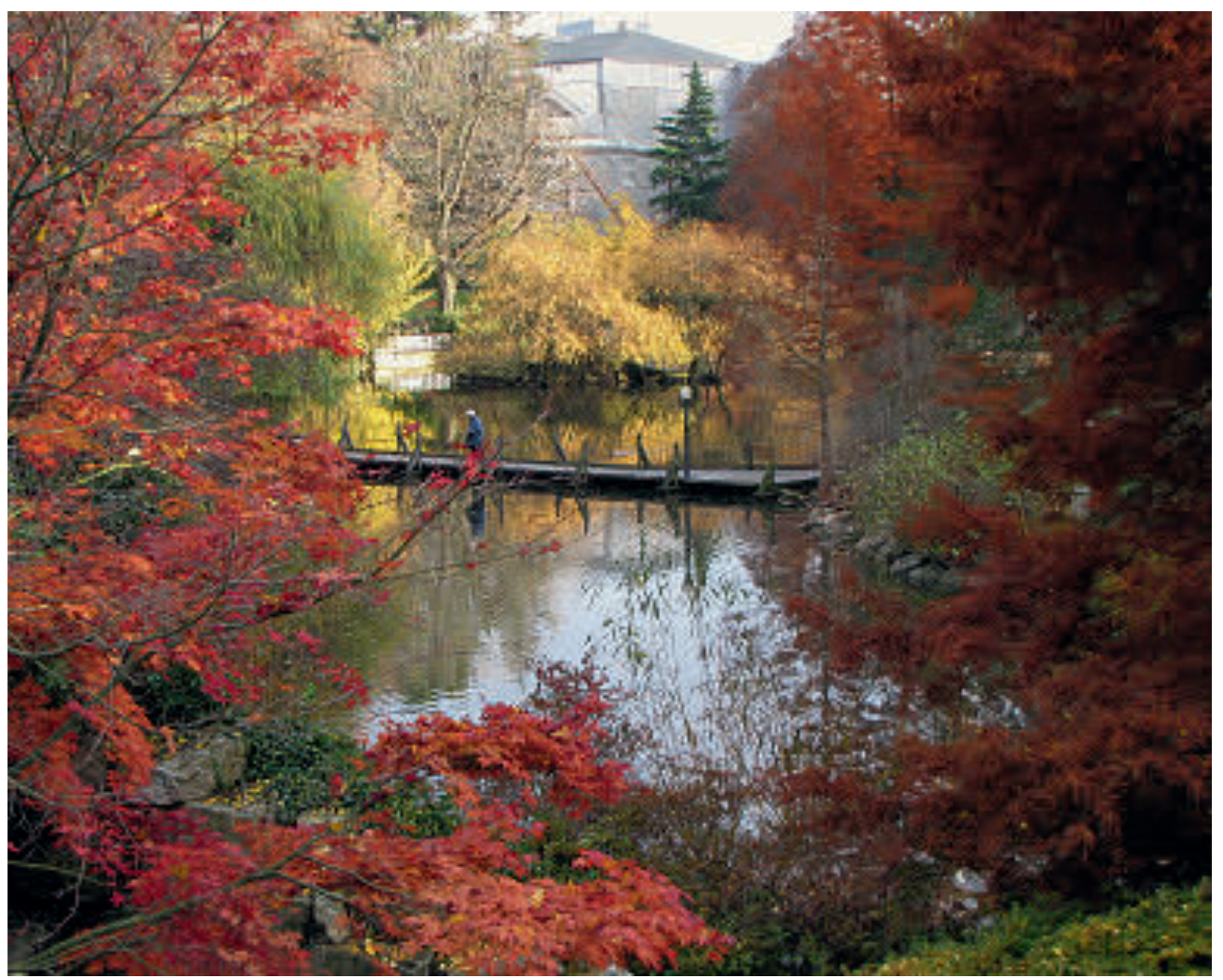

worden war. Als Vorbild diente die Hängebrücke im Parc des Buttes Chaumont in Paris. Dieser 25 ha große Landschaftspark wurde 1867 zur Pariser Weltausstellung unter NAPOLEON III. eröffnet. Der aufwendig gestaltete, in einem Steinbruch nahe einer Müllkippe gelegene Park erregte damals international viel Aufmerksamkeit und erhielt durchweg großes Lob. Gartenarchitekten aus der zweiten Hälfte des

Abb. 8 (Seite 68 oben) : Blick über den Bootsweiher, Juni 2015.

Abb. 9 (Seite 68 unten): Blick vom Schweizerhaus über die Hängebrücke und den Weiher auf das Gesellschaftshaus auf einer alten Ansichtskarte (abgeschickt am 23.3.1905).

Abb. 10 (oben): Herbstlicher Blick vom ehemaligen Standort des Schweizerhauses über den Bootsweiher mit Brücke und das von großen, alten Bäumen umgebene Gesellschaftshaus, Oktober 2010.
19. Jahrhunderts wollten nur das Beste vom Besten schaffen und ließen sich von besonders gelungenen zeitgenössischen Parks und Gärten inspirieren: der vielgepriesene Parc des Buttes Chaumont lieferte nicht nur Heinrich SiesMAYER für den Palmengarten, sondern auch den Erschaffern des Türkenschanzparks in Wien wertvolle Anregungen. Die Brücke wurde im Jahr 1931 unter Max Bromme wegen Baufälligkeit entfernt und durch Trittsteine, später durch einen Holzsteg ersetzt, der noch heute existiert. Die zwei Mauern an den beiden Zugängen des heutigen Stegs sind die Reste der ehemaligen Brückenköpfe.

\section{Wasserfall}

Im Zuge einer Modernisierung wurde zu Beginn der 1930er-Jahre auch der Steingarten neu gestaltet. Dazu war Albert Baylli beauftragt worden. Weichen musste dafür auch der 




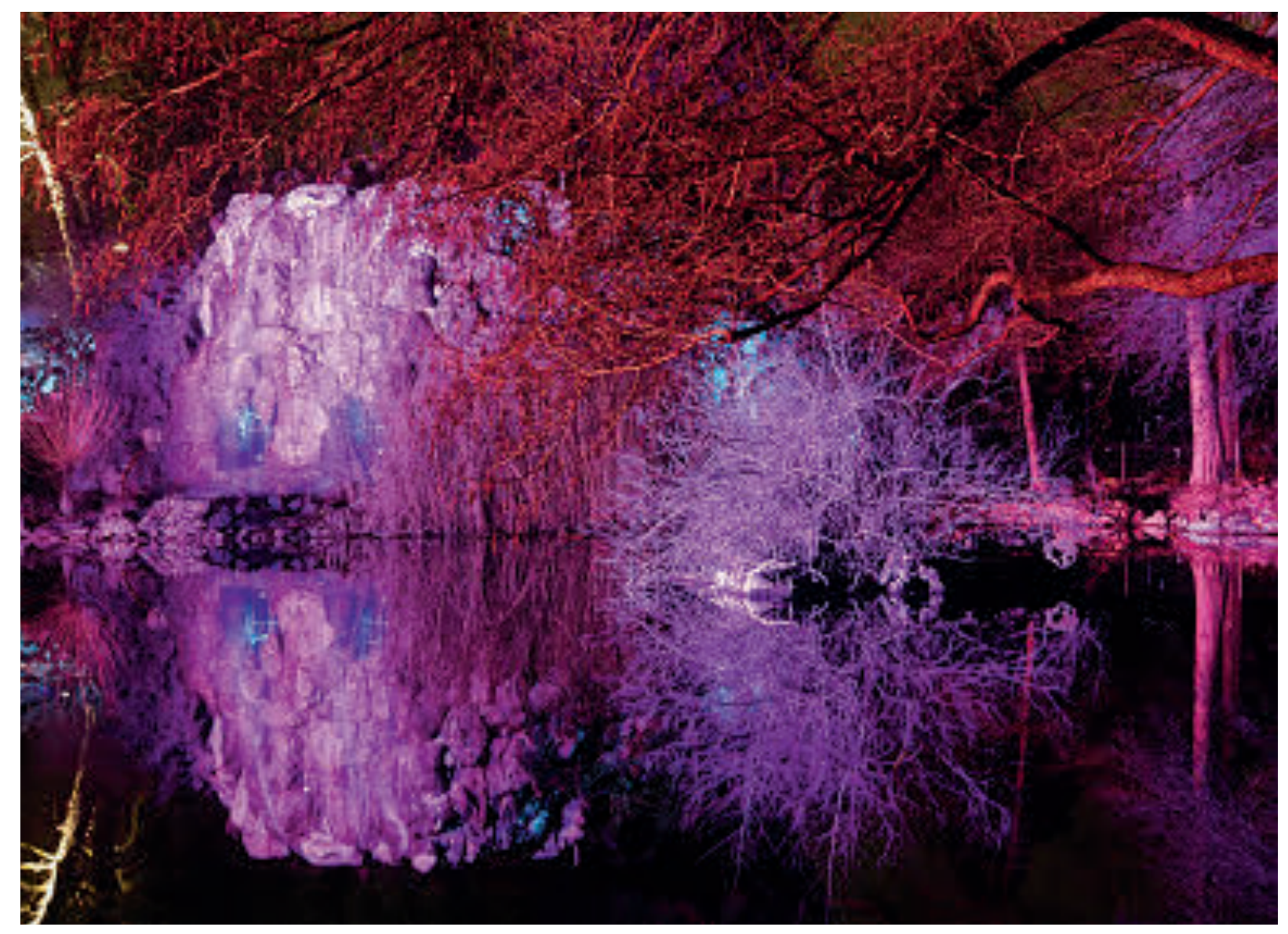

Wasserfall. Erst 1971 wurde er wiederhergestellt und 1986 erneut überarbeitet. Heute ist er wieder ein besonderer Blickfang. Besonders schön ist auch ein Blick durch die Fenster der Grotte auf den Wasserfall. In Wintern mit starkem Frost, in denen das Wasser nicht abgestellt wird, vereist der Wasserfall und es bilden sich zahlreiche Eiszapfen. In den Wintermonaten sind Steingarten, Grotte und Wasserfall auch Highlights der Veranstaltung „Winterlichter", die seit 2012 in den Wintermonaten stattfindet. Dann ist das Ensemble märchenhaft illuminiert und erweckt erneut romantische Gefühle.
Abb. 11 (Seite 70 oben): Statt der alten Hängebrücke führt ein Holzsteg über den Weiher, Juni 2015.

Abb. 12 (Seite 70 unten links): Brückenkopf der Hängebrücke auf einer alten Ansichtskarte.

Abb. 13 (Seite 70 unten rechts): Die Mauern an den Enden des Holzsteges sind die Reste der Brückenköpfe, Juni 2015.

Abb. 14 (oben): Illumination der Grotte während der „Winterlichter“, Dezember 2013. 